\title{
THE CERN SPS EXPERIMENT ON MICROWAVE TRANSMISSION THROUGH THE BEAM PIPE
}

\author{
T. Kroyer ${ }^{1 \dagger}$, F. Caspers, E. Mahner, CERN, Geneva, Switzerland \\ $\dagger$ TU Wien, Institute of Communications and Radio-Frequency Engineering
}

\begin{abstract}
In the CERN SPS microwave transmission measurements through beampipe sections with a length of $30 \mathrm{~m}$ and $7 \mathrm{~m}$ respectively have been carried out in the frequency range 2-4 GHz since spring 2003. Here we report on new results obtained with improved measurement techniques during the 2004 run. Observation techniques include a fast real time scope, spectrum analyser IF and video output signal registration and baseband signal observation using a PC soundcard. The unexpected beam-induced amplitude modulation has been confirmed on all kinds of available beams including single bunches. It was found that there is a correlation between the amount of beam induced signal attenuation and the beam losses registered by external scintillators. Potential theoretical models are discussed.
\end{abstract}

\section{INTRODUCTION}

During the 2003 and 2004 run in CERN's SPS, a series of microwave transmission measurements were made to determine whether there is an interaction between the expected electron cloud and microwaves propagating as waveguide modes in the beam pipe. Theoretical models predict a phase shift as well as an attenuation of the electromagnetic wave [1]. Any such change is modulated at the SPS revolution frequency of about $43 \mathrm{kHz}$, which translates phase and amplitude changes into PM and AM, making highly sensitive sideband measurements possible. A measurement method based on such an effect could potentially determine the electron cloud density integrated over the measurement track. This would be interesting, since it allows an in-situ measurement in existing accelerators at relatively little cost. This method can also be tried in LHC, where measurements over an entire arc are possible using the coupling structures of the LHC reflectometer [2]. In the SPS, the measurements were performed mainly using the fundamental $\mathrm{TE}_{11}$ and the $\mathrm{TE}_{31}$ waveguide mode with cutoff at about 1 and $3 \mathrm{GHz}$ in the flat dipole chamber. Other modes were avoided by limiting the frequency range and by selective excitation. Two sections of beam pipe were used, one $\approx 30 \mathrm{~m}$ long arc section (long track) in the beam direction and in $\mathrm{a} \approx 7 \mathrm{~m}$ straight part (short track) in the opposite direction. On the long track there are one focusing quadrupole, three main bending magnets, one sextupole and one correction dipole, on the short track there are only one skew quad and one correction dipole.

\footnotetext{
${ }^{1}$ tom.kroyer@cern.ch
}

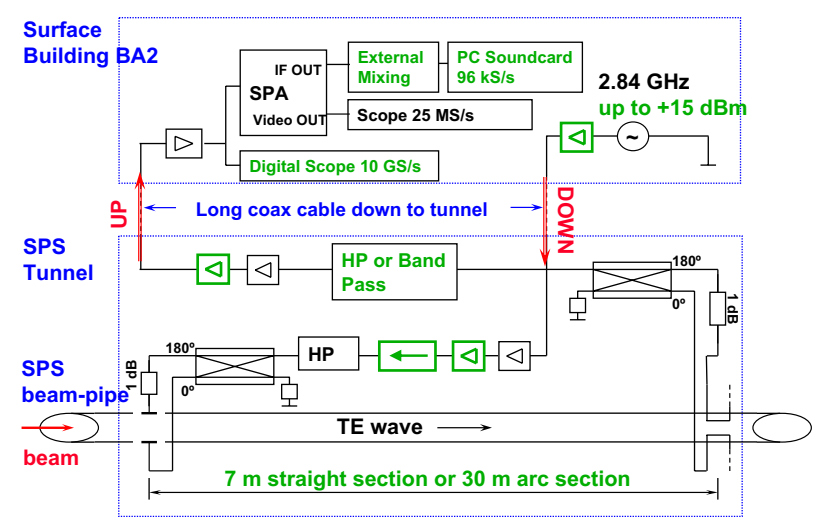

Figure 1: Measurement set-up 2004. The green elements were added since the 2003 run. The amplifiers sketched with thick green lines can be used up to about $+28 \mathrm{dBm}$ output power.

\section{MEASUREMENT SET-UP}

The main results from the 2003 run have been reported elsewhere [3]. After the lessons learned, the measurement set-up as well as the data processing techniques were considerably refined. Fig. 1 shows the set-up used at the end of the 2004 run. The main changes and extension were

- Adding the $7 \mathrm{~m}$ short straight track to check the influence of bending magnets

- Use of power amplifiers to increase dynamic range

- Installation of additional filters in the up-going signal paths and an isolator in the down-going path to reduce the impact of beam-induced signals.

On the data acquisition and processing side a fast digital scope (up to $20 \mathrm{GS} / \mathrm{s}$ ) and long trace acquisition (entire machine cycles) using downmixing to base-band and an external PC soundcard were used.

Testing of the measurement set-up In order to get a grip on possible measurement errors, the entire set-up was thoroughly studied. The $1 \mathrm{~dB}$ compression point of the amplifiers was measured to be able to evaluate saturation effects.

In the down-going path saturation was avoided by adjusting the $\mathrm{CW}$ signal on the surface. However, it cannot be excluded beforehand that the power from the beam incident on the output stage of the last amplifier drives it into saturation. Up to $4 \mathrm{GHz}$, the beam signal rejection by the high 
pass and the isolator is of the order of $40 \mathrm{~dB}$, so this possibility must be checked. Indeed, in a lab experiment it was found that the effective amplifier gain decreases slightly $(\approx$ $0.2 \mathrm{~dB}$ ) for a $10 \mathrm{dBm} \mathrm{CW}$ signal in the $1 \mathrm{GHz}$ range incident on its output.

The "up" (return) path is inherently more sensitive to beaminduced signals, since it cannot be protected by an isolator (Fig. 1). A $2 \mathrm{GHz}$ cut-off waveguide filter and a 2.8 to $3.2 \mathrm{GHz}$ band-pass filter were used for the long track and the short track, respectively. Since the beam has still a considerable power density in this spectral region, a certain impact on the amplifier gain can be expected. Intermodulation measurements were performed in the lab in order to quantify the gain compression in the presence of beam-induced signals. Driven with a CW signal at its maximum specified input level of $+10 \mathrm{dBm}$, the power amplifier used (Minicircuits ZHL-42) showed a gain compression of about $0.3 \mathrm{~dB}$ when an AM modulated spurious signal with a power of 3 $\mathrm{dBm}$ was applied. Even when the latter is very low (-30 $\mathrm{dBm}$ ), a small gain compression can still be noticed due to the small non-linearity of the amplifier. In the frequency domain, a typical AM spectrum appears around the carrier. This means that intermodulation due to beam signals can significantly alter the $\mathrm{CW}$ transmission through the amplifier chain.

\section{RESULTS}

In the data taken during the 2003 and 2004 runs, phenomena were found that can be grouped in five categories.

- Attenuation with beam. For all beams and most carrier frequencies, attenuation was observed. In the time domain the carrier was downconverted by a spectrum analyser (SPA) running in zero-span mode centered on the carrier with about $3 \mathrm{MHz}$ IF bandwidth. The video output signal of the SPA was plotted in time domain. When the beam passes, there is a distinct drop in amplitude. Depending on beam type, long or short track and carrier frequency, this drop ranges between fractions of a dB to $30 \mathrm{~dB}$. Correspondingly, in the frequency domain an AM spectrum can be found.

- Strong absorption peaks and periodicities after each injection. For many beams, the first modulation sideband tracked over the entire machine cycle showed strong peaks after each injection and in general increasing absorption as the magnets are ramped. These signals show some correlation with beam losses, but above all they are strongly correlated with the beam spectrum. This suggests that intermodulation in the amplifiers of the up-going path has a significant impact on the measurement.

For the absorption peaks just after injection, a 3-turn periodicity and an overlaid $1000 \mathrm{~Hz}$ structure could be resolved during roughly the first $200 \mathrm{~ms}$.

- Asymmetric spectrum at cyclotron resonance. When the cyclotron resonance condition for electrons

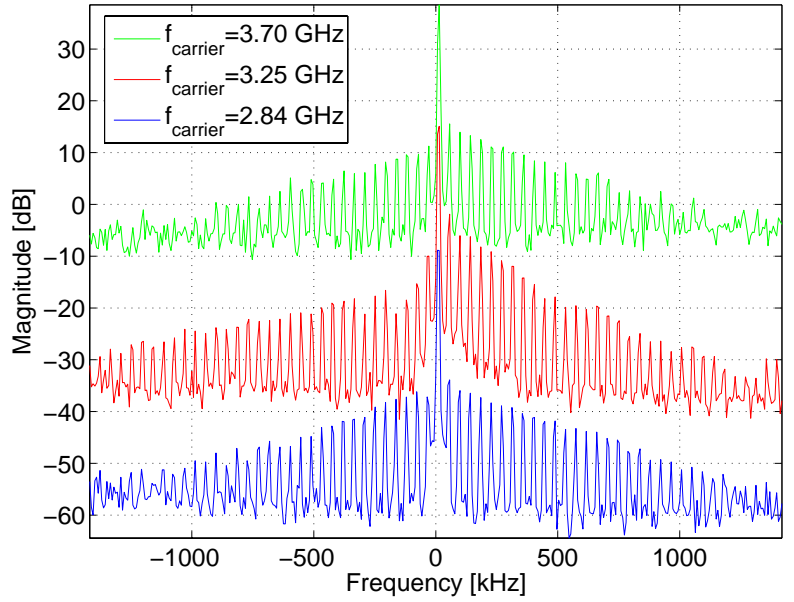

Figure 2: Modulation spectrum in the region of the electron cyclotron resonance at the injection flat bottom $\left(f_{\text {res }} \approx\right.$ $3.25 \mathrm{GHz}$ ). Spectra stacked vertically for clarity.

(charge $e$, mass $m$ )

$$
\omega=\frac{e B_{0}}{m}
$$

is fulfilled in the main bending magnets at the SPS injection flat bottom $\left(f_{\text {carrier }} \approx 3.25 \mathrm{GHz}\right.$ for $B_{0}=$ $0.117 \mathrm{~T}$ ), a visibly asymmetric modulation spectrum can be found, pointing to a superposition of AM and PM. In fig. 2 the spectra with carrier frequencies in the region of the cyclotron resonance are compared.

- Tails. For certain beams, especially "strong" LHC type beams, absorption still occurred after the passage of the beam with decay times of the order of $1 \mu \mathrm{s}$, as reported in [3]. The origin of these erratic tails is still not fully understood.

- Stored beams. For measurements with stored bunched beams it was found that after the ramping, the attenuation decreases slowly and the modulation spectrum becomes asymmetric.

\section{Bench cross-check}

In order to demonstrate the fact that there is a significant interaction between a magnetized electron cloud and waveguide modes, a bench measurement was set up. An electron gun was used to inject electrons of up to $500 \mathrm{eV}$ energy laterally into a circular cross-section tube. At the location where the electrons enter the tube, a static magnetic field was applied with a small magnet. The propagation of waveguide modes was then measured as a function of electron energy, current and external magnetic field. Even though the magnetic field was too small to allow operation at cyclotron resonance, a very small but reproducible amplitude change with respect to the reference measurement without magnetic field was found when the electron energy was increased from 10 to $500 \mathrm{eV}$. A set-up with higher magnetic field to study the behaviour at cyclotron resonance is currently under way. 


\section{DISCUSSION}

It turned out that in the SPS we are dealing with a very complex situation; a localized strongly time dependent electron plasma in a magnetic field. First interpretations started from the interaction of microwaves in free space with a homogeneous plasma. This would simply lead to a phase shift of the electromagnetic wave, for a length L, given by

$$
\Delta \phi=-\frac{1}{2} \frac{\omega_{p}^{2}}{\omega c} L
$$

with the plasma frequency $\omega_{p}=\sqrt{4 \pi \rho_{e} r_{e} c^{2}}$, the classical electron radius $r_{e}$ and the speed of light $c$. However, the fact that the electromagnetic waves are guided and the existence of an inhomogeneous magnetic field seriously complicates the situation. To our knowledge, there is presently no numerical tool available that could easily simulate such a system. Various approaches were tried in order to explain the observed phenomena.

The drop in carrier signal when the beam is passing may be partially due to saturation of the amplifiers. However, the strongly asymmetric spectrum at cyclotron resonance cannot be explained this way. The beam-induced signals do not depend on the actual carrier frequency used, so saturation effects should not change the observed spectrum. Apart from the main dipoles, the quadrupoles and other magnets are also expected to house an electron cloud. In these elements the electric field of the TE wave is not parallel to the static magnetic field, which implies that a strong interaction between electrons and the electromagnetic wave can be expected. Two possible energy loss mechanisms for the waveguide mode are absorption and mode conversion. Work is going on in order to get a quantitative estimation. The 3-turn periodicity after injection is apparently related to the vertical tune. The beam-induced signals get stronger for an off-center beam, and with a vertical fractional tune close to 0.16 the beam induced signals will drive the amplifiers closer towards saturation about every third turn. The overlaid $1000 \mathrm{~Hz}$ symmetry is simply due to the beating of the tune and the revolution frequency.

For the erratic tails, lab tests have been done showing that amplifier saturation cannot be responsible for this effect, since the amplifier recovery time after heavy overloads is in the range of $1 \mathrm{~ns}$.

\section{Lessons learned}

During the last run, lots of experience was gained with respect to instrumentation and data acquisition. The following points have to be addressed in order to reduce parasitic effects.

- Suppress the beam-induced signals as much as possible in both the down and the up-going signal path. A waveguide cavity filter with a few $\mathrm{MHz}$ bandwidth could be used to this end.

- Choose straight sections of beam pipe without magnets or an arc section with dipoles only. Except for fringe fields the wave E field is then parallel to the static B field, making an interpretation of the results easier.

- Work with a carrier frequency a factor three or so above the highest electron cyclotron frequency. This should considerably reduce cyclotron absorption [4], making it easier to measure the expected phase shifts. In the LHC at injection energy, the cyclotron resonance is below $1 \mathrm{GHz}$, which makes it possible to meet this condition using the fundamental TE or TM waveguide mode.

\section{CONCLUSION}

After improving the measurement and data acquisition techniques before the 2004 run, lots of new data was taken. All the main results from 2003, especially the microwave signal attenuation with beam and the fluctuating tails could be repeated. However, it was discovered that amplifier saturation plays a bigger role than previously expected. Repeating the experiment with special precautions to reduce saturation effects as much as possible would thus be highly desirable. Coming to the theoretical explanations, cyclotron resonance effects in the dipoles and other magnets now appear to be responsible for the interaction between the electromagnetic wave and the electron plasma. In particular the asymmetric modulation spectrum at cyclotron resonance in the main dipoles is a strong indication for such an effect. However, due to the complexity of the problem, no quantitative estimations are available yet.

\section{ACKNOWLEDGEMENTS}

We would like to thank Thomas Bohl, Frank Zimmermann and Stefano Alberti for help and advice. Thanks to Flemming Pedersen for inspiring discussion, Trevor Linnecar for support, Miguel Jimenez and Jean-Francois Malo for help with the experiment.

\section{REFERENCES}

[1] Heald, M. A. and Wharton, C. B., Plasma Diagnostics with Microwaves, Wiley, New York, 1965

[2] Caspers, F; Kroyer, T Coupler Structures for the LHC Beam Pipe Waveguide Mode Reflectometer, CERN-LHC-ProjectReport-764, Geneva, 2004

[3] Kroyer, T. et alias, Unexpected Results on Microwave Waveguide Mode Transmission Measurements in the SPS Beam Pipe, Proceedings of the 31st ICFA Advanced Beam Dynamics Workshop (ECLOUD '04), CERN-2005-001, 2005

[4] Prater, R. Heating and current drive by electron cyclotron wave, Physics of Plasmas, Volume 11, Number 5, San Diego, 2004 